\title{
Combination of galectin-3, CK19 and HBME-1 immunostaining improves the diagnosis of thyroid cancer
}

\author{
VANESSA ARCOLIA ${ }^{1}$, FABRICE JOURNE $^{1,2}$, FLORENCE RENAUD $^{3}$, EMMANUELLE LETEURTRE $^{3}$, \\ HANS-JOACHIM GABIUS ${ }^{4}$, MYRIAM REMMELINK ${ }^{5}$ and SVEN SAUSSEZ ${ }^{1,6}$
}

\begin{abstract}
${ }^{1}$ Laboratory of Human Anatomy and Experimental Oncology, Faculty of Medicine and Pharmacy, University of Mons, B-7000 Mons; ${ }^{2}$ Laboratory of Oncology and Experimental Surgery, Jules Bordet Institute, Free University of Brussels, B-1000 Brussels, Belgium; ${ }^{3}$ Lille University, UMR-S 1172, JPARC, Jean-Pierre Aubert Research Center, F-59000 Lille, France; ${ }^{4}$ Institute of Physiological Chemistry, Faculty of Veterinary Medicine, Ludwig-Maximilians-University, D-80539 Munich, Germany; ${ }^{5}$ Department of Pathology, Erasme Hospital, Free University of Brussels, B-1070 Brussels; ${ }^{6}$ Department of Oto-Rhino-Laryngology, CHU Saint-Pierre, Free University of Brussels, B-1000 Brussels, Belgium
\end{abstract}

Received January 17, 2017; Accepted June 26, 2017

DOI: $10.3892 / \mathrm{ol} .2017 .6719$

\begin{abstract}
Currently, fine-needle aspiration is the most frequently used pre-operative technique for diagnosis of malignant thyroid tumors, however, pathologists are unable to reach efficient and accurate differential diagnoses between benign and malignant thyroid nodules. To aid in resolving this issue, immunohistochemistry for galectins (gal)-1, -3, -7, -8, cytokeratin 19 (CK19), Hector Battifora Mesothelial Epitope-1 (HBME-1) and thyroid peroxidase (TPO) was performed on two tissue microarrays composed of 66 follicular adenomas (FA) and 66 papillary carcinomas (PC). The identification of optimal cut-off levels and the diagnostic value of single immunomarkers or combinations were evaluated using the receiver operating characteristic curve analysis. Signal intensities for gal-1, gal-3, CK19 and HBME-1 were significantly greater in PC compared with FA $(\mathrm{P}<0.001)$. Conversely, expression levels of TPO were significantly increased in FA compared with PC ( $\mathrm{P}<0.001)$. Gal-3 and CK19 appeared to be the most sensitive markers (97 and 98\%, respectively), whereas galectin-1 was the most specific (97\%). The combination of gal-3, CK19 and HBME-1 acted as the most efficient and informative marker panel reaching the greatest specificity (97\%) and sensitivity (95\%) for the diagnosis of PCs. The findings suggest that this combination of markers may improve the reliability of diagnosis of thyroid cancer.
\end{abstract}

Correspondence to: Professor Sven Saussez, Laboratory of Human Anatomy and Experimental Oncology, Faculty of Medicine and Pharmacy, University of Mons, Pentagone 2A, 6 Avenue du Champ de Mars, B-7000 Mons, Belgium

E-mail: sven.saussez@umons.ac.be

Key words: cytokeratin, galectin, lectin, malignancy, ROC curve

\section{Introduction}

The estimated occurrence of palpable thyroid nodules in the general population ranges from 4 to $7 \%$ (1). While thyroid nodules are therefore rather common, thyroid cancers are rare. Thyroid ultrasonography and thyroid fine-needle aspiration (FNA) are frequently used preoperative techniques to diagnose malignant thyroid tumors. However, FNA leads to indeterminate biopsy results in 10-20\% of all cases, when solely based on cytopathological evaluation $(2,3)$.

Nowadays, the preoperative characterization of thyroid nodules remains a challenge for clinicians. To address this issue ever increasing numbers of studies had evaluated the value of immunohistochemical markers such as galectin (gal)-3, cytokeratin 19 (CK19), Hector Battifora Mesothelial-1 (HBME-1) and thyroid peroxidase (TPO) (4-14). Although some are promising markers to distinguish benign from malignant thyroid lesions, none is individually reliable for differential diagnosis. Actually, each marker has its limitations because of significant expression in benign thyroid nodules to a notable extent (15-20). Therefore, novel immunohistochemical markers or combinations are required to define criteria for distinction between benign and malignant thyroid lesions, especially regarding the classification of thyroid follicular lesions.

Homodimeric gal- 1 is a potential new candidate because its expression has been shown to be upregulated in cancers (21-24), including thyroid carcinoma (25-27). Proteomic profiling has also suggested gal-1 to be a potential biomarker of thyroid cancer $(28,29)$. Furthermore, our team has previously reported for the first time a high serum level of both gal-1 and gal-3 in patients diagnosed with benign thyroid lesions and well-differentiated thyroid carcinoma compared to healthy individuals (30). In addition, having revealed functional additivity and antagonism between members of the galectin family $(31,32)$, we take steps to network analysis by co-monitoring expression of gal-7 and gal-8.

In the present study, we compared the diagnostic value of gal-1, gal-3, CK19, HBME-1 and TPO, alone and in combination, 
in benign and malignant thyroid lesions in order to determine the usefulness of each marker or a combination of markers, allowing the most accurate diagnosis of thyroid cancer through preoperative assessment of nodular thyroid lesions.

\section{Materials and methods}

Clinical data. The immunohistochemical detection of gal-1, gal-3, gal-7, and gal-8, CK19, HBME-1 and TPO was studied in two tissue microarrays (TMA) composed of 66 follicular adenomas (FA) and 66 papillary carcinomas (PC). The available population data were gender, age and histopathologic features. This clinical series included 100 women and 32 men. The mean age of patients with FA was 44 years (range, 13-76 years) and 41 years for patients with PC (range, 9-73 years) (33). The patient samples and clinical data were retrieved from the records of the Lille University Hospital (Lille, France) between August 2000 and September 2001, selected and analysed by two pathologists (Professor E. Leteurtre and Dr F. Renaud). No inclusion and exclusion criteria of the selected patients were used in the current study. Written informed consent was obtained from all the patients to use the surgical specimens for scientific research.

Immunohistochemistry and histopathologic examination. Specimens were fixed in $10 \%$ buffered formalin and paraffin-embedded. Five-micrometer sections were stained with hematoxylin and eosin for examination by light microscopy. The entire paraffin-embedded blocks were selected and arrayed in triplicate $0.6 \mathrm{~mm}$ tissue cores for TMA construction (Beecher Instruments, Silver Springs, MD, USA). The $5 \mu \mathrm{m}$-thick tissue sections were deparaffinized and heat pretreated (citrate buffer or EDTA; Ventana Medical Systems, Tucson, AZ, USA) before incubation with i) specific primary antibody against gal-1 (polyclonal rabbit anti-human galectin-1, 1:100) and gal-3 (polyclonal rabbit anti-human galectin-3, 1:200) (34,35), gal-7 (polyclonal rabbit anti-human galectin-7, 1:50) and gal-8 (polyclonal rabbit anti-human galectin-8, 1:20) (36,37), all anti-galectin antibodies rigorously controlled against occurrence of cross-reactivity to human galectins and depleted by respective cycles of affinity chromatography if positive, and CK19 (monoclonal mouse anti-human cytokeratin-19, 1:50; M0772; Dako, Glostrup, Denmark), HBME-1 (monoclonal mouse anti-human HBME-1, 1:100; M3505; Dako), TPO (monoclonal mouse anti-human TPO, 1:50; TPO47; Biocytex, Marseille, France); then ii) corresponding biotinylated secondary antibody (760-500, ultraView Universal DAB Detection kit; Ventana Medical Systems); and finally iii) avidin-biotin-peroxidase complex (ABC kit; Vector Laboratories, Burlingame, CA, USA). The slides were thoroughly washed with PBS between each incubation step. Immunocomplexes were finally visualized by exposure to the diaminobenzidine chromogen (DAB; BioGenex, Fremont, CA, USA) in the presence of $\mathrm{H}_{2} \mathrm{O}_{2}$. After rinsing, the sections were counterstained with luxol fast blue and mounted with a synthetic medium. To exclude antigen-independent staining, the incubation step with primary antibodies was omitted from the protocol as negative controls. In all cases, these controls were negative (data not shown). The specificity of gal-1 and gal-3 antibodies was validated by western blotting in different thyroid cancer cell lines (B-CPAP, FTC133C and 8505C cell lines derived from papillary, follicular and anaplastic thyroid carcinoma respectively) reporting immunoreactive band for gal-1 at $14 \mathrm{kDa}$ and for gal-3 at $26 \mathrm{kDa}$ (data not shown). The FTC133C and 8505C cell lines were analyzed to confirm the absence of mycoplasma contamination and the presence of characteristic markers of follicular thyroid carcinoma and anaplastic thyroid carcinoma, respectively. Both cell lines have been characterized at the IRIBHM laboratory (Professor C. Maenhaut, ULB, Brussels, Belgium) (38). A blind semi-quantitative analysis of immunostainings was independently performed by three pathologists (Professor E. Leteurtre, Dr F. Renaud, Professor M. Remmelink) using a light microscope (Axiocam MRc5; Carl Zeiss, Hallbergmoos, Germany). Each tissue specimen was scored (0-6) by adding the percent of immunopositive cells (range, $0-3$ : $0=0 ; 1=1-33$; $2=34-66$ and $3=67-100 \%$ ) to the staining intensity (range, $0-3$ : $0=$ none; $1=$ low; $2=$ moderate and $3=$ high). The overall score used for subsequent statistical analysis was the mean of three spots of the same tumor.

Statistical analysis. Groups of data were compared using the non-parametric Mann-Whitney test. The diagnostic performances of single or combined immunomarkers and the identification of the optimal cut-off points for the diagnosis of malignancy were evaluated using the receiver operating characteristic (ROC) curves and the assessment of the area under the ROC curve (AUC). The specificity, sensitivity, positive predictive value (PPV) and negative predictive value (NPV) of markers, alone or combined, were evaluated from crosstabs based on cut-off points and significance were calculated using the Fisher's exact test. P-value $<0.05$ was considered as significant. Statistical analyses were performed with IBM SPSS Statistics 23 (IBM, Ehningen, Germany).

\section{Results}

Gal-1/-3/-7/-8, CK19, HBME-1 and TPO immunostaining profiles in benign and malignant thyroid lesions. The first aim of our study was to assess the expression levels of seven markers (gal-1, gal-3, gal-7, gal-8, TPO, CK19 and HBME-1) by immunohistochemistry in two series of TMAs composed of 66 cases of FA and 66 cases of PC.

In sections of PCs, CK19 and HBME-1 staining was either cytoplasmic or both cytoplasmic and apical, with intensity increase at the apical membrane (Fig. 1B and C). Signal presence for gal-1 and gal-3 was ubiquitous, both in tumor cell (cytoplasmic and nuclear staining) and the stroma associated to cancers and adenomas (Fig. 1A and D). The expression pattern of TPO showed that staining was cytoplasmic, relatively intense at the apical membrane of benign cells (Fig. 1E).

The statistical analysis of the results of the cytoplasmic immunostaining revealed that the level expression of the four markers (gal-1, gal-3, CK19 and HBME-1) was significantly higher in cancer cells of PC compared to epithelial cells in FA $(\mathrm{P}<0.001$, Mann-Whitney test) (Fig. 1A-D). By contrast, the cytoplasmic expression of TPO is higher in adenomas than in cancers $(\mathrm{P}<0.001$, Mann-Whitney test) (Fig. 1E).

Of note, staining levels of gal-7 and gal-8 were nuclear and cytoplasmic both in FA and PC without any statistical 
A

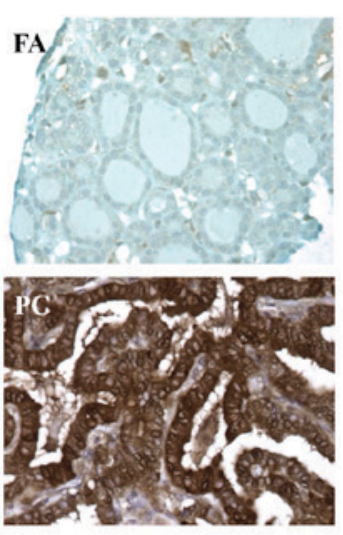

C

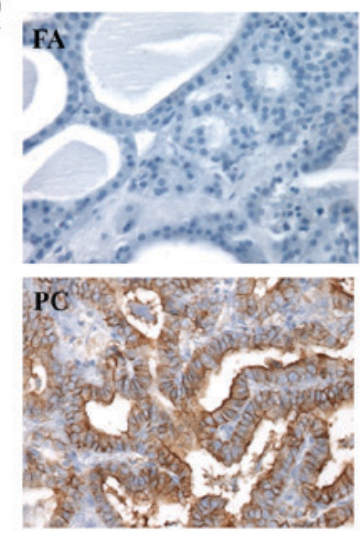

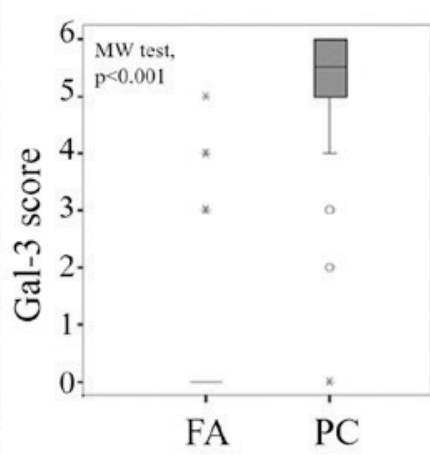

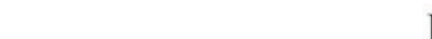

B
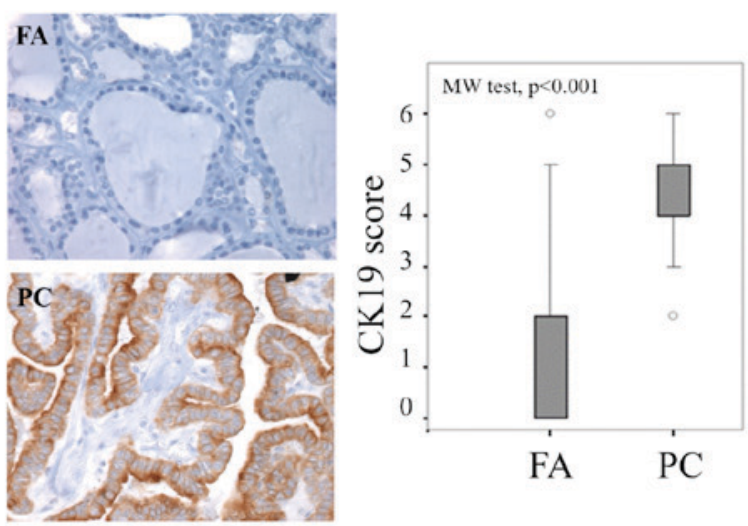

D
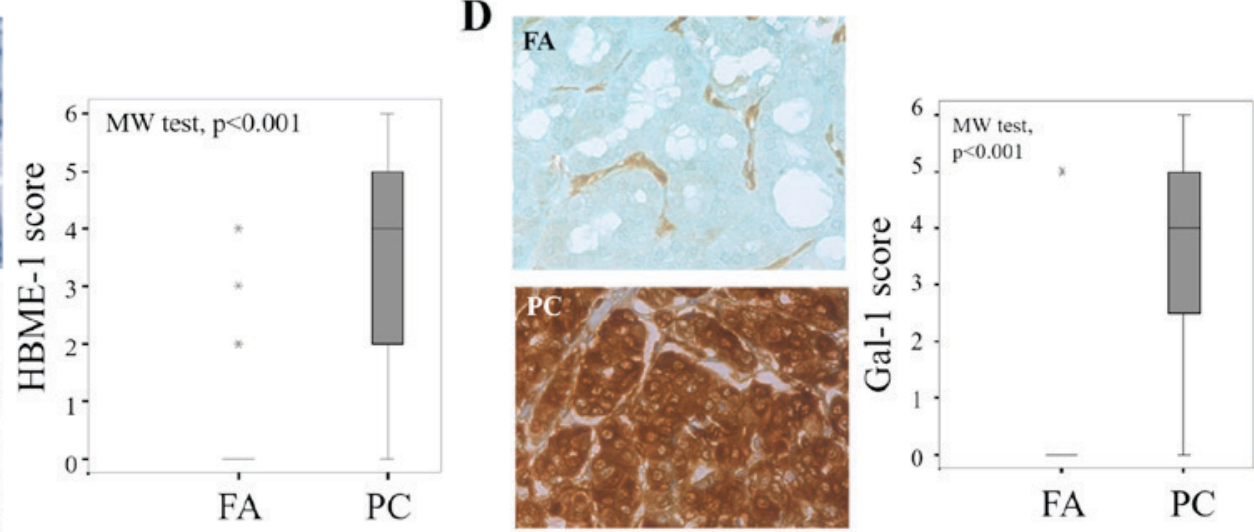

E

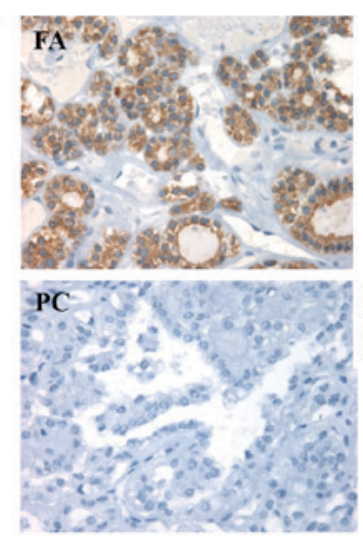

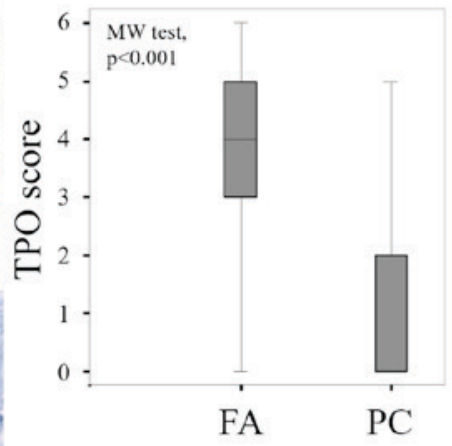

Figure 1. Evaluation of gal-3, CK19, HBME-1, gal-1 and TPO expression in benign and malignant thyroid lesions. Immunohistochemical evaluation of expression and statistical analysis of (A) gal-3, (B) CK19, (C) HBME-1, (D) gal-1 and (E) TPO in tissue microarray composed by FA and PC (P<0.001, Mann-Whitney test). Magnification, $\mathrm{x} 400$. The immunostaining was semi-quantitatively assessed in the cytoplasmic compartment and scored from 0 to 6 by summing staining intensity (0-3) and percentage of positivity (0-3). Data are presented as box plots indicating the 1 st and the 3 rd quartiles centered on medians (thick lines) with whiskers for the minimum and maximum non-outlier values, the 'o' symbols are outliers and the ${ }^{\text {*, }}$ symbols show the extreme values. Gal-3, galectin-3; CK19, cytokeratin 19; HBME-1, Hector Battifora Mesothelial Epitope-1; TPO, thyroid peroxidase; FA, follicular adenoma; PC, papillary carcinoma.

difference between cytoplasmic immunostaining in each group $(\mathrm{P}=0.12$ and $\mathrm{P}=0.47$, Mann-Whitney test, respectively) (Fig. 2).

Gal-1 was completely absent in the epithelial compartment on almost all benign thyroid neoplasms (Fig. 1D), whereas a weak to moderate signal was found in benign samples for gal-3, CK19 and HBME-1 (Fig. 1A, B and C, respectively), suggesting that monitoring gal-1 could be more reliable to distinguish malignant from benign lesions.

Diagnostic performances of individual or combined immunomarkers. For each individual marker, the cut-off has been defined from the ROC curve (Fig. 3A). The cut-offs allowing to separate negative/low vs. positive immunostaining were $>0$ for gal-1, gal-3 and HBME-1, and >2 for CK19 and TPO (Table I and Fig. 1). The diagnostic performance of the markers was individually evaluated by comparing areas under the ROC. As described in Table I, the area under the ROC curve of gal-3 (AUC $=0.957)$ is greater than the area under the ROC curves of the CK19 (AUC=0.947), HBME-1 (AUC=0.910), gal-1 $(\mathrm{AUC}=0.883)$ and TPO (AUC=0.879). From crosstab analyses, gal-3 and CK19 appear to be the most sensitive markers (97 and 98\%, respectively), while gal-1 is the most specific one $(97 \%)(\mathrm{P}<0.001$, Fisher's exact test). HBME-1 and TPO 
$\mathbf{A}$

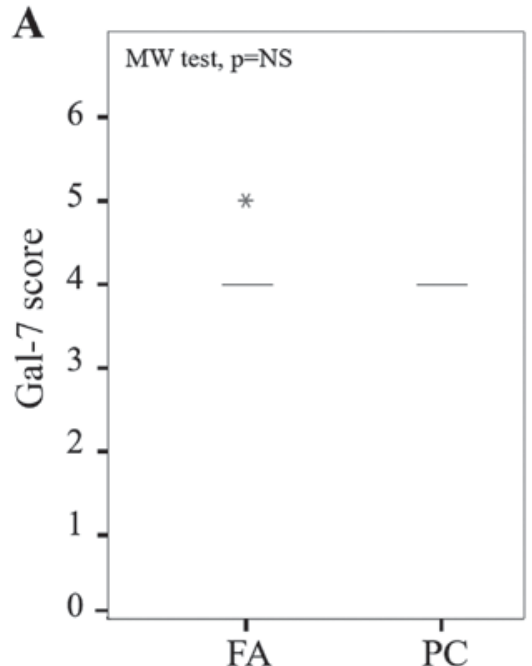

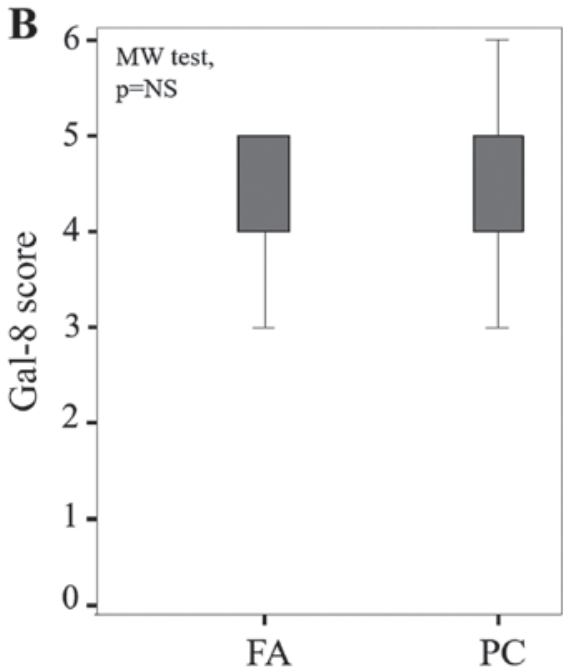

Figure 2. Evaluation of gal-7 and gal-8 expression in FA and PC. Statistical analysis of (A) gal-7 and (B) gal-8 in a series of 66 cases of FA and 66 cases of PCs ( $\mathrm{P}=\mathrm{NS}$, Mann-Whitney test). The immunostaining was semi-quantitatively assessed in the cytoplasmic compartment and scored from 0 to 6 by summing staining intensity (0-3) and percentage of positivity (0-3). Data are presented as box plots indicating the 1st and the 3rd quartiles centered on medians (thick lines) with whiskers for the minimum and maximum non-outlier values, the ${ }^{* *}$ symbol shows the extreme values. Gal-7, galectin-7; FA, follicular adenoma; PC, papillary carcinoma.

A

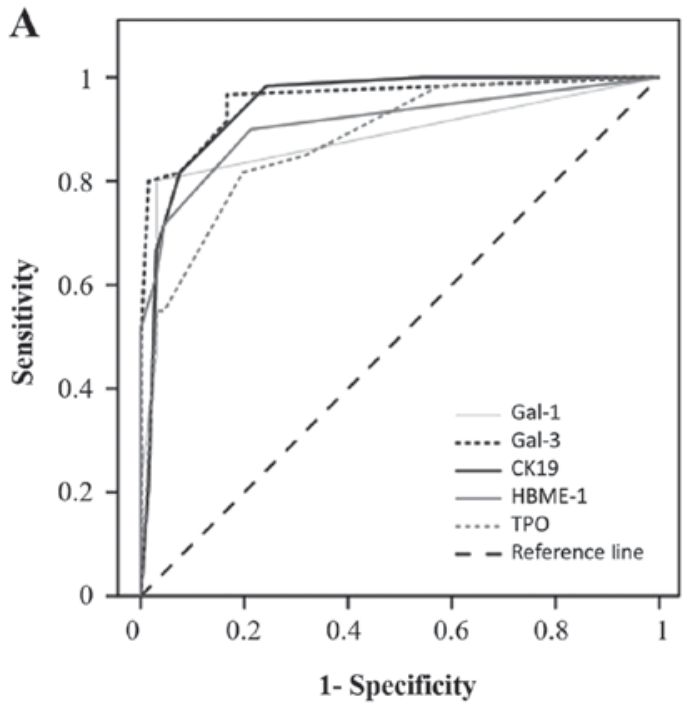

B

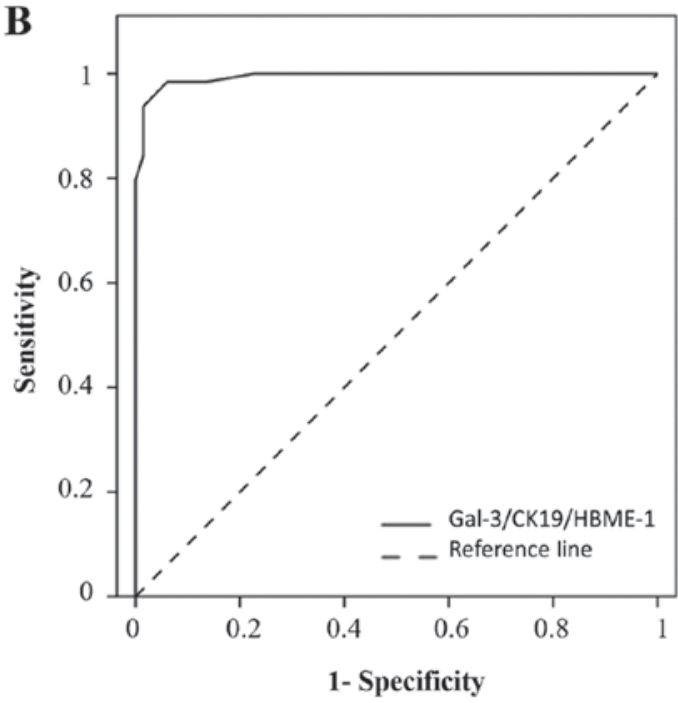

Figure 3. Receiver operating characteristic curves of single and combined thyroid markers. (A) ROC curve for positive immunocytochemistry expression of gal-1, gal-3, CK19, HBME-1 and TPO in the diagnosis of PCs. Areas under the curve: Gal-3=0.957; CK19=0.047; HBME-1=0.910; gal-1=0.883; TPO=0.879. (B) ROC curve for positive immunohistochemistry expression of the combination of three markers (gal-3/CK19/HBME-1) in the diagnosis of PCs. Area under the curve, 0.994. ROC, receiver operating characteristic; gal-1, galectin-1; CK19, cytokeratin 19; HBME-1, Hector Battifora Mesothelial Epitope-1; TPO, thyroid peroxidase; PC, papillary carcinoma.

exhibited good sensitivities (88 and $83 \%$, respectively) and good specificities ( 79 and $80 \%$, respectively) (Table I).

Statistical analysis for all possible permutations using the five markers to discriminate between FA and PC was shown in Table II. Of note, as TPO is a negative diagnostic marker for cancer, we used its inverse score (6-score) to combine it to others, and mean scores (0-6) were calculated for all combinations. The diagnostic performance of the combination of markers was evaluated by comparing areas under the ROC curves. The data revealed that the combination of gal-3/CK19/HBME-1 exhibits the highest performance to assess the diagnosis of malignancy (AUC $=0.994$, cut-off $>2.5$ ) (Table II and Fig. 3B). The sensitivity, specificity, PPV and NPV were also calculated for the panel of combined markers. Several combinations of markers such as gal-3/CK19, gal-3/CK19/HBME-1,gal-3/CK19/gal-1,gal-3/HBME-1/gal-1/TPOor gal-3/CK19/HBME-1/gal-1/TPO improves specificity (>95\%) and sensitivity $(>90 \%)$. However, based on these calculations, the combination of gal-3/CK19/HBME-1 is the best one, associating high sensitivity (95\%) and high specificity (97\%) for the diagnosis of $\mathrm{PC}(\mathrm{P}<0.001$, Fisher's exact test) (Table II).

\section{Discussion}

Although conventional histology and FNA are considered as gold standards, the pathologists are confronted with difficulties 
Table I. Diagnostic value of individual markers to distinguish malignant from benign thyroid tumors.

\begin{tabular}{lccccccc}
\hline Markers & AUC & Cut-off & Spe $(\%)$ & Sens $(\%)$ & PPV (\%) & NPV (\%) & Fisher's exact test $\left(10^{-15}\right)$ \\
\hline Galectin-3 & 0.957 & $>0$ & 83 & 97 & 85 & 96 & $\mathrm{P}=1.07$ \\
CK19 & 0.947 & $>2$ & 76 & 98 & 80 & 98 & $\mathrm{P}=1.02$ \\
HBME-1 & 0.910 & $>0$ & 79 & 88 & 81 & 87 & $\mathrm{P}=4.20$ \\
Galectin-1 & 0.883 & $>0$ & 97 & 80 & 96 & 83 & $\mathrm{P}=5.16$ \\
TPO & 0.879 & $>2$ & 80 & 83 & 80 & 83 & $\mathrm{P}=345.47$ \\
\hline
\end{tabular}

AUC, area under the curve; Spe, specificity; Sens, sensitivity; PPV, positive predictive value; NPV, negative predictive value; CK19, cytokeratin 19; HBME-1, Hector Battifora Mesothelial Epitope-1; TPO, thyroid peroxidase.

Table II. Diagnostic value of combined markers in discrimination of malignant from benign thyroid tumors.

\begin{tabular}{|c|c|c|c|c|c|c|c|}
\hline Markers & AUC & Cut-off & Spe $(\%)$ & Sens $(\%)$ & $\operatorname{PPV}(\%)$ & $\operatorname{NPV}(\%)$ & Fisher's exact test $\left(10^{-15}\right)$ \\
\hline Gal-3/CK19/HBME-1 & 0.994 & $>2.5$ & 97 & 95 & 97 & 96 & $\mathrm{P}=1.57$ \\
\hline Gal-3/CK19/HBME-1/gal-1 & 0.991 & $>2$ & 95 & 95 & 95 & 95 & $\mathrm{P}=1.57$ \\
\hline Gal-3/CK19/HBME-1/TPO & 0.991 & $>2.5$ & 97 & 90 & 97 & 91 & $\mathrm{P}=16.73$ \\
\hline Gal-3/CK19/HBME-1/gal-1/TPO & 0.989 & $>2.5$ & 97 & 91 & 96 & 91 & $\mathrm{P}=4.63$ \\
\hline Gal-3/CK19 & 0.988 & $>3$ & 99 & 92 & 98 & 93 & $\mathrm{P}=5.48$ \\
\hline Gal-3/HBME-1/TPO & 0.986 & $>2.5$ & 94 & 87 & 93 & 88 & $\mathrm{P}=16.73$ \\
\hline Gal-3/CK19/gal-1 & 0.985 & $>2$ & 97 & 92 & 97 & 93 & $\mathrm{P}=8.91$ \\
\hline Gal-3/HBME-1 & 0.985 & $>2$ & 95 & 95 & 95 & 95 & $\mathrm{P}=8.29$ \\
\hline Gal-3/CK19/TPO & 0.985 & $>2.5$ & 92 & 94 & 92 & 91 & $\mathrm{P}=4.71$ \\
\hline Gal-3/HBME-1/gal-1/TPO & 0.985 & $>2$ & 97 & 91 & 95 & 91 & $\mathrm{P}=1.01$ \\
\hline Gal-3/HBME-1/gal-1 & 0.984 & $>2$ & 95 & 87 & 95 & 89 & $P=6.18$ \\
\hline Gal-3/CK19/gal-1/TPO & 0.984 & $>2$ & 94 & 95 & 94 & 95 & $\mathrm{P}=1.50$ \\
\hline CK19/HBME-1/gal-1 & 0.982 & $>2$ & 94 & 91 & 94 & 91 & $\mathrm{P}=7.06$ \\
\hline Gal-3/TPO & 0.979 & $>2.5$ & 92 & 90 & 92 & 91 & $\mathrm{P}=4.42$ \\
\hline Gal-3/gal-1/TPO & 0.979 & $>2$ & 94 & 82 & 93 & 84 & $\mathrm{P}=12.86$ \\
\hline CK19/HBME-1/gal-1/TPO & 0.979 & $>2$ & 91 & 90 & 90 & 91 & $\mathrm{P}=22.82$ \\
\hline CK19/HBME-1 & 0.972 & $>2$ & 89 & 91 & 90 & 91 & $\mathrm{P}=14.85$ \\
\hline CK19/gal-1 & 0.972 & $>2$ & 95 & 92 & 95 & 93 & $\mathrm{P}=7.06$ \\
\hline CK19/gal-1/TPO & 0.969 & $\geq 2.5$ & 94 & 90 & 93 & 91 & $\mathrm{P}=20.51$ \\
\hline HBME-1/gal-1/TPO & 0.966 & $>2$ & 95 & 85 & 95 & 87 & $\mathrm{P}=12.30$ \\
\hline CK19/HBME-1/TPO & 0.966 & $>3$ & 95 & 87 & 95 & 86 & $\mathrm{P}=7.68$ \\
\hline HBME-1/gal-1 & 0.965 & $>2$ & 97 & 80 & 96 & 83 & $P=5.16$ \\
\hline Gal-3/gal-1 & 0.957 & $>0$ & 83 & 97 & 85 & 96 & $\mathrm{P}=11.13$ \\
\hline CK19/TPO & 0.949 & $>3$ & 84 & 86 & 84 & 85 & $P=14.29$ \\
\hline Gal-1/TPO & 0.942 & $>2$ & 95 & 84 & 95 & 86 & $\mathrm{P}=1.91$ \\
\hline HBME-1/TPO & 0.936 & $>2.5$ & 92 & 86 & 92 & 97 & $\mathrm{P}=1.47$ \\
\hline
\end{tabular}

AUC, area under the curve; Spe, specificity; Sens, sensitivity; PPV, positive predictive value; NPV, negative predictive value; gal-3. galectin-3; CK19, cytokeratin 19; HBME-1, Hector Battifora Mesothelial Epitope-1; TPO, thyroid peroxidase.

in reaching an accurate differential diagnosis between benign and malignant thyroid nodules. To improve disease identification, immunohistochemical markers, such as gal-3, CK19, HBME-1 and TPO, have been proposed and their efficiencies for thyroid cancer diagnosis have been evaluated. CK19 is the smallest member of cytokeratin family responsible for the structural integrity of epithelial cells. Several studies reported that CK19 expression is intense and diffuse in PC and absent or low in benign thyroid lesions $(5,6,12-14)$. Gal-3, a structurally unique member of galectin family with an $\mathrm{N}$-terminal tail composed of nine collagen-like repeats $(39,40)$, is associated with the pathogenesis of well-differentiated thyroid carcinoma (4,7-11). HBME-1 is a surface antigen localized in the microvilli of the mesothelial cells (41). It had a wider expression in PC compared to follicular carcinomas and FA $(42,43)$. TPO is a membrane enzyme involved in the synthesis of thyroid 
hormones (44). By contrast, it has been shown to be relevant in the diagnosis of FAs. Its labeling is negative in carcinomas regardless of their histopathologic status (papillary, follicular, medullary or anaplastic) $(45,46)$.

However, the value of clinical use of these markers is controversial, because positivity was also reported in benign cases (13-20,41,42). Mehrotra et al showed that gal-3 was expressed in a large proportion of FAs, multinodular goiters and Hashimoto's thyroiditis (16). In the study of Mataraci et al, CK19 expression was found in adenomatous nodular hyperplasia and FA (13). Furthermore, a focal positive labeling may exist in FAs and goiter for HBME-1 $(41,42)$. In addition, a study by Weber et al reported to TPO a $50 \%$ sensitivity for diagnosis of PC, suggesting that TPO should be combined with other markers such as gal-3 (47).

Thus, the current challenge is to find new immunohistochemical markers that might be more helpful to refine differential diagnosis between benign and malignant. Widely studied in other types of cancers, gal-1 remained poorly documented in the thyroid pathologies (26-28). Because of that, one of the main contribution of this study to advance the status of the field was to determine whether gal-1 may be viewed as a complementary biomarker for diagnosis of thyroid cancer assessing nodular lesions. In this context, we studied the diagnostic performance of galectin-1 individually and in combination with four other thyroid tumor markers used in clinical practice. Our data showed that the expression of this galectin is significantly higher in PC than in FA. As a single marker, gal-1 displayed a higher specificity (97\%) than gal-3 and CK19 which showed higher sensitivity (97 and 98\%, respectively). Of note, comparing to the serum levels of both gal-1 and gal-3 (30), our current study on tissues revealed higher values for the sensitivity and specificity. Gal-1, Gal-3 and CK19 can be used in association to improve discrimination between malignant and benign thyroid neoplasms. So, when we combined two to five markers, we significantly improved the specificity, the sensitivity as well as the PPVs/NPVs for malignancy. The association of positivity for gal-3, CK19 and HBME-1 proved to be the most relevant combination in the distinction between PCs and FAs. Hence, our data advocate the concept of the use of combinations of immunomarkers in clinical practice to diagnose thyroid carcinomas. Evidently, a panel of markers might be more helpful than the use of a single one to improve diagnostic accuracy $(20,48-51)$. Of relevance for the galectin network (52), our data indicate that different members of this family have non-redundant distribution profiles, indicating non-overlapping functional spectra. Galectins are widely distributed in a tissue- and cell-specific manner. This is particularly emphized for gal-7 and gal-8 which are not differentially expressed between FA and PC, suggesting that these galectins are not involved in thyroid carcinogenesis or that transformation to tumor cells did not impact their expressions.

In summary, the diagnostic problems in thyroid pathology are still present in many laboratories and this paper can be potentially useful for improving information. In this study, we used TMAs to test a panel of markers that also include gal-1, gal-7 and gal-8. We demonstrated that gal-1 is a useful immunohistochemical marker to discriminate malignant tumors from benign thyroid nodules. Our observations further validate that gal-3 is a sensitive marker for the diagnosis of thyroid malignancy, and we add support for its combination with CK19 and HBME-1 with the highest performance for the diagnosis of well-differentiated thyroid cancer. Such combination of markers should be validated in a larger series of tissues including various subtypes of thyroid lesions.

\section{Acknowledgements}

The study received financial support from the University of Mons (Mons, Belgium). We thank Dr Köhrle (Institute of Experimental Endocrinology of the Charité, Humboldt University, Berlin, Germany) and Professor C. Maenhout (IRIBHM ULB, Brussels, Belgium) for providing the FTC133C and 8505C cell lines (derived from follicular and anaplastic thyroid carcinoma respectively).

\section{References}

1. Hegedüs L: Thyroid ultrasonography as a screening tool for thyroid disease. Thyroid 14: 879-880, 2004.

2. Gharib H and Papini E: Thyroid nodules: Clinical importance, assessment and treatment. Endocrinol Metab Clin North Am 36: 707-735, vi, 2007.

3. Williams ED: Guest editorial: Two proposals regarding the terminology of thyroid tumors. Int J Surg Pathol 8: 181-183, 2000.

4. Liu Z, Li X, Shi L, Maimaiti Y, Chen T, Li Z, Wang S, Xiong Y, Guo H, He W, et al: Cytokeratin 19, thyroperoxidase, HBME-1 and galectin-3 in evaluation of aggressive behavior of papillary thyroid carcinoma. Int J Clin Exp Med 7: 2304-2308, 2014.

5. Flanagan JN, Pineda P, Knapp PE, De Las Morenas A, Lee SL and Braverman LE: Expression of cytokeratin 19 in the diagnosis of thyroid papillary carcinoma by quantitative polymerase chain reaction. Endocr Pract 14: 168-174, 2008.

6. Krzeslak A, Gaj Z, Pomorski L and Lipinska A: Expression of cytokeratin 19 in the cytosolic fraction of thyroid lesions: ELISA and western blot analysis. Mol Med Rep 1: 565-569, 2008.

7. Sumana BS, Shashidhar S and Shivarudrappa AS: Galectin-3 immunohistochemical expression in thyroid neoplasms. J Clin Diagn Res 9: EC07-EC11, 2015.

8. Bartolazzi A, Gasbarri A, Papotti M, Bussolati G, Lucante T, Khan A, Inohara H, Marandino F, Orlandi F, Nardi F, et al: Application of an immunodiagnostic method for improving preoperative diagnosis of nodular thyroid lesions. Lancet 357 : 1644-1650, 2001

9. Inohara H, Honjo Y, Yoshii T, Akahani S, Yoshida J, Hattori K, Okamoto S, Sawada T, Raz A and Kubo T: Expression of galectin-3 in fine-needle aspirates as a diagnostic marker differentiating benign from malignant thyroid neoplasms. Cancer 85: 2475-2484, 1999.

10. Gasbarri A, Martegani MP, Del Prete F, Lucante T, Natali PG and Bartolazzi A: Galectin-3 and CD44v6 isoforms in the preoperative evaluation of thyroid nodules. J Clin Oncol 17: 3494-3502, 1999.

11. Carpi A, Rossi G, Coscio GD, Iervasi G, Nicolini A, Carpi F, Mechanick JI and Bartolazzi A: Galectin-3 detection on large-needle aspiration biopsy improves preoperative selection of thyroid nodules: A prospective cohort study. Ann Med 42: 70-78, 2010.

12. Liu Z, Yu P, Xiong Y, Zeng W, Li X, Maiaiti Y, Wang S, Song H, Shi L, Liu C, et al: Significance of CK19, TPO, and HBME-1 expression for diagnosis of papillary thyroid carcinoma. Int $\mathbf{J}$ Clin Exp Med 8: 4369-4374, 2015.

13. Mataraci EA, Ozgüven BY and Kabukçuoglu F: Expression of cytokeratin 19, HBME-1 and galectin-3 in neoplastic and nonneoplastic thyroid lesions. Pol J Pathol 63: 58-64, 2012.

14. Schmitt AC, Cohen C and Siddiqui MT: Paired box gene 8 , HBME-1, and cytokeratin 19 expression in preoperative fine-needle aspiration of papillary thyroid carcinoma: Diagnostic utility. Cancer Cytopathol 118: 196-202, 2010.

15. Niedziela M, Maceluch J and Korman E: Galectin-3 is not an universal marker of malignancy in thyroid nodular disease in children and adolescents. J Clin Endocrinol Metab 87: 4411-4415, 2002 . 
16. Mehrotra P, Okpokam A, Bouhaidar R, Johnson SJ, Wilson JA, Davies BR and Lennard TW: Galectin-3 does not reliably distinguish benign from malignant thyroid neoplasms. Histopathology 45 : 493-500, 2004

17. Mills LJ, Poller DN and Yiangou C: Galectin-3 is not useful in thyroid FNA. Cytopathology 16: 132-138, 2005.

18. Park YJ, Kwak SH, Kim DC, Kim H, Choe G, Park DJ, Jang HC, Park SH, Cho BY and Park SY: Diagnostic value of galectin-3, HBME-1, cytokeratin 19, high molecular weight cytokeratin, cyclin D1 and p27(kip1) in the differential diagnosis of thyroid nodules. J Korean Med Sci 22: 621-628, 2007.

19. Zhu X, Sun T, Lu H, Zhou X, Lu Y, Cai X and Zhu X: Diagnostic significance of CK19, RET, galectin-3 and HBME-1 expression for papillary thyroid carcinoma. J Clin Pathol 63: 786-789, 2010.

20. Barroeta JE, Baloch ZW, Lal P, Pasha TL, Zhang PJ and LiVolsi VA: Diagnostic value of differential expression of CK19, Galectin-3, HBME-1, ERK, RET and p16 in benign and malignant follicular-derived lesions of the thyroid: An immunohistochemical tissue microarray analysis. Endocr Pathol 17: 225-234, 2006

21. Danguy A, Camby I and Kiss R: Galectins and cancer. Biochim Biophys Acta 1572: 285-293, 2002.

22. Demydenko D and Berest I: Expression of galectin-1 in malignant tumors. Exp Oncol 31: 74-79, 2009.

23. Balan V, Nangia-Makker P and Raz A: Galectins as cancer biomarkers. Cancers (Basel) 2: 592-610, 2010.

24. Smetana K Jr, André S, Kaltner H, Kopitz J and Gabius HJ: Context-dependent multifunctionality of galectin-1: A challenge for defining the lectin as therapeutic target. Expert Opin Ther Targets 17: 379-392, 2013.

25. Chiariotti L, Berlingieri MT, Battaglia C, Benvenuto G, Martelli ML, Salvatore P, Chiappetta G, Bruni CB and Fusco A: Expression of galectin-1 in normal human thyroid gland and in differentiated and poorly differentiated thyroid tumors. Int J Cancer 64: 171-175, 1995.

26. Xu XC, el-Naggar AK and Lotan R: Differential expression of galectin-1 and galectin-3 in thyroid tumors. Potential diagnostic implications. Am J Pathol 147: 815-822, 1995.

27. Salajegheh A, Dolan-Evans E, Sullivan E, Irani S, Rahman MA, Vosgha H, Gopalan V, Smith RA and Lam AK: The expression profiles of the galectin gene family in primary and metastatic papillary thyroid carcinoma with particular emphasis on galectin-1 and galectin-3 expression. Exp Mol Pathol 96: 212-218, 2014.

28. Torres-Cabala C, Bibbo M, Panizo-Santos A, Barazi H, Krutzsch H, Roberts DD and Merino MJ: Proteomic identification of new biomarkers and application in thyroid cytology. Acta Cytol 50: 518-528, 2006.

29. Paron I, D'Ambrosio C, Scaloni A, Berlingieri MT, Pallante PL, Fusco A, Bivi N, Tell G and Damante G: A differential proteomic approach to identify proteins associated with thyroid cell transformation. J Mol Endocrinol 34: 199-207, 2005.

30. Saussez S, Glinoer D, Chantrain G, Pattou F, Carnaille B, André S, Gabius HJ and Laurent G: Serum galectin-1 and galectin-3 levels in benign and malignant nodular thyroid disease. Thyroid 18 : 705-712, 2008

31. Sanchez-Ruderisch H, Fischer C, Detjen KM, Welzel M, Wimmel A, Manning JC, André S and Gabius HJ: Tumor suppressor p16 INK4a: Downregulation of galectin-3, an endogenous competitor of the pro-anoikis effector galectin-1, in a pancreatic carcinoma model. FEBS J 277: 3552-3563, 2010

32. Weinmann D, Schlangen K, André S, Schmidt S, Walzer SM Kubista B, Windhager R, Toegel S and Gabius HJ: Galectin-3 induces a pro-degradative/inflammatory gene signature in human chondrocytes, teaming up with galectin-1 in osteoarthritis pathogenesis. Sci Rep 6: 39112, 2016.

33. Verhulst P, Devos P, Aubert S, Buob D, Cranshaw I, Do Cao C, Pattou F, Carnaille B, Wemeau JL and Leteurtre E: A score based on microscopic criteria proposed for analysis of papillary carcinoma of the thyroid. Virchows Arch 452: 233-240, 2008.

34. Toegel S, Bieder D, André S, Kayser K, Walzer SM, Hobusch G, Windhager R and Gabius HJ: Human osteoarthritic knee cartilage: Fingerprinting of adhesion/growth-regulatory galectins in vitro and in situ indicates differential upregulation in severe degeneration. Histochem Cell Biol 142: 373-388, 2014

35. Kaltner H, Seyrek K, Heck A, Sinowatz F and Gabius HJ: Galectin-1 and galectin-3 in fetal development of bovine respiratory and digestive tracts. Comparison of cell type-specific expression profiles and subcellular localization. Cell Tissue Res 307: 35-46, 2002
36. Langbein S, Brade J, Badawi JK, Hatzinger M, Kaltner $\mathrm{H}$, Lensch M, Specht K, André S, Brinck U, Alken P and Gabius HJ: Gene-expression signature of adhesion/growth-regulatory tissue lectins (galectins) in transitional cell cancer and its prognostic relevance. Histopathology 51: 681-690, 2007.

37. Danguy A, Rorive S, Decaestecker C, Bronckart Y, Kaltner H, Hadari YR, Goren R, Zich Y, Petein M, Salmon I, et al: Immunohistochemical profile of galectin-8 expression in benign and malignant tumors of epithelial, mesenchymatous and adipous origins, and of the nervous system. Histol Histopathol 16: 861-868, 2001

38. Saiselet M, Floor S, Tarabichi M, Dom G, Hébrant A, van Staveren WC and Maenhaut C: Thyroid cancer cell lines: An overview. Front Endocrinol (Lausanne) 3: 133, 2012.

39. Kopitz J, Vértesy S, André S, Fiedler S, Schnölzer M and Gabius HJ: Human chimera-type galectin-3: Defining the critical tail length for high-affinity glycoprotein/cell surface binding and functional competition with galectin-1 in neuroblastoma cell growth regulation. Biochimie 104: 90-99, 2014.

40. Ippel H, Miller MC, Vértesy S, Zheng Y, Cañada FJ, Suylen D, Umemoto K, Romanò C, Hackeng T, Tai G, et al: Intra- and intermolecular interactions of human galectin-3: Assessment by full-assignment-based NMR. Glycobiology 26: 888-903, 2016.

41. Rigau V, Martel B, Evrard C, Rousselot P and Galateau-Salle F: HBME-1 immunostaining in thyroid pathology. Ann Pathol 21: 15-20, 2001 (In French).

42. Prasad ML, Pellegata NS, Huang Y, Nagaraja HN, de la Chapelle A and Kloos RT: Galectin-3, fibronectin-1, CITED-1, HBME1 and cytokeratin-19 immunohistochemistry is useful for the differential diagnosis of thyroid tumors. Mod Pathol 18: 48-57, 2005.

43. Liu YY, Morreau H, Kievit J, Romijn JA, Carrasco N and Smit JW: Combined immunostaining with galectin-3, fibronectin-1, CITED-1, Hector Battifora mesothelial-1, cytokeratin-19, peroxisome proliferator-activated receptor-\{gamma $\}$, and sodium/iodide symporter antibodies for the differential diagnosis of non-medullary thyroid carcinoma. Eur J Endocrinol 158: 375-384, 2008.

44. De Micco C, Savchenko V, Giorgi R, Sebag F and Henry JF Utility of malignancy markers in fine-needle aspiration cytology of thyroid nodules: Comparison of Hector Battifora mesothelial antigen-1, thyroid peroxidase and dipeptidyl aminopeptidase IV. Br J Cancer 98: 818-823, 2008.

45. De Micco C, Ruf J, Chrestian MA, Gros N, Henry JF and Carayon P: Immunohistochemical study of thyroid peroxidase in normal, hyperplastic, and neoplastic human thyroid tissues. Cancer 67: 3036-3041, 1991.

46. Faggiano A, Caillou B, Lacroix L, Talbot M, Filetti S, Bidart JM and Schlumberger M: Functional characterization of human thyroid tissue with immunohistochemistry. Thyroid 17: 203-211, 2007.

47. Weber KB, Shroyer KR, Heinz DE, Nawaz S, Said MS and Haugen BR: The use of a combination of galectin-3 and thyroid peroxidase for the diagnosis and prognosis of thyroid cancer. Am J Clin Pathol 122: 524-531, 2004.

48. de Matos LL, Del Giglio AB, Matsubayashi CO, de Lima Farah M, Del Giglio A and da Silva Pinhal MA: Expression of CK-19, galectin-3 and HBME-1 in the differentiation of thyroid lesions: Systematic review and diagnostic meta-analysis. Diagn Pathol 7: 97, 2012

49. Dunđerović D, Lipkovski JM, Boričic I, Soldatović I, Božic V, Cvejić D and Tatić S: Defining the value of CD56, CK19, Galectin 3 and HBME-1 in diagnosis of follicular cell derived lesions of thyroid with systematic review of literature. Diagn Pathol 10: 196, 2015

50. de Matos PS, Ferreira AP, de Oliveira Facuri F, Assumpção LV, Metze K and Ward LS: Usefulness of HBME-1, cytokeratin 19 and galectin-3 immunostaining in the diagnosis of thyroid malignancy. Histopathology 47: 391-401, 2005.

51. Cheung CC, Ezzat S, Freeman JL, Rosen IB and Asa SL: Immunohistochemical diagnosis of papillary thyroid carcinoma. Mod Pathol 14: 338-342, 2001.

52. Kaltner H, Toegel S, Caballero GG, Manning JC, Ledeen RW and Gabius HJ: Galectins: Their network and roles in immunity/tumor growth control. Histochem Cell Biol 147: 239-256, 2017. 